\section{CBRNE Trauma: A Unified Approach to Clinical Management}

\section{D.J. Baker, MPbil, DM, FRCA, FRSM}

Professor, Consultant Anesthesiologist and Medical Toxicologist, UK

Chemical, biological, radiological, and nuclear hazards currently are grouped together in terms of management of releases and casualties. Explosive hazards recently have been added to this grouping to include hazards from the deliberate use of improvised explosive devices (IEDs). The chemical, biological, radiological, nuclear, and explosive (CBRNE) classification provides a focus for planning, early and effective emergency responses, and emphasizes the inherent secondary dangers to responders and the need for protection. It also provides a controlled, operational framework for the early provision of life support and antidote responses during disasters.

Although the CBRNE grouping has provided common ground for planners, management of the incident, and the provision of emergency medical care, it must be remembered that the nature of the individual CBRNE hazards is very different in terms of immediate effects on victims and the latency with which they are revealed. There are clear, apparent differences in the early management of exposure to a chemical risk compared with the trauma caused by an IED. However, in reality, there are identifiable common pathways of injury from CBRNE hazards which determine both immediate and continuing medical care. For example, blunt and penetrating trauma from blast, together with injury to the lungs has a final pathophysiological pathway of cell injury through initial hypovolaemia, shock, tissue hypoxia, and multiple organ failure. Toxic trauma from chemical and biological agents may have different initial stages, but can lead to a similar clinical result with common pathophysiological end stages.

In recent years, much has been learned from intensive care management of conventional and toxic trauma about appropriate methods of life support, particularly in ventilation strategies. This presentation will review similarities between conventional and toxic trauma from CBRNE hazards and will consider what early life support strategies may be appropriate to alter the course of subsequent clinical development. Keywords: chemical, biological, radiological, nuclear, and explosive (CBRNE); life support; management; responders; trauma Prehosp Disaster Med

\section{Attitude of Physicians Toward Chemical Warfare Casualty Management}

Amir Krivoy;, ${ }^{1,2}$ Shai Shrot, ${ }^{1,3}$ Yossi Rosman; ${ }^{1,3}$

Erez Ramati; ${ }^{1}$ Ram Sagi ${ }^{1}$

1. CBRN Medicine Branch, Medical Corps, Israel Defense Forces, Israel

2. Sackler Faculty of Medicine, Tel-Aviv University, Israel

3. Sheba Health Center, Tel-Hashomer, Israel

Introduction: Chemical warfare agents (CWAs) still are an immanent threat on the military and civilian population. The use of CWAs in wartime or as a terrorist weapon was demonstrated in recent years, and resulted in numerous physical and psychological casualties. and a grave psychological impact. The Tokyo subway sarin attack on 1995 exemplified the vast impact of chemical terrorism in general and the use of CWAs as a terrorist weapon. ${ }^{1}$ This event was the trigger for many countries and health authorities to establish preparedness programs to mitigate such an event. As part of the medical management of such an event, medical teams are expected to encounter CWA casualties, in the field or in hospitals. ${ }^{2}$ However, most physicians are not prepared or educated for the specific toxidromes and treatment protocols of CWA poisoning. ${ }^{3}$ Moreover, the psychological implications of coping with CWA casualties also should be considered, meaning that these scenarios are perceived as unique, and differ from the regular trauma casualties these medical teams are used to manage. ${ }^{4} \mathrm{~A}$ survey indicated that whereas $80 \%$ of physician respondents were willing to participate in the diagnosis and treatment of bioterrorism cases, only $21 \%$ believed that they were prepared to handle the cases. ${ }^{5}$

Methods: Military physicians, on regular duty or reserve, who participated in the mandatory CBRN medicine course during their training at the military medical school. Regarding attitude toward Chemical Warfare Casualties Medical Treatment (CWCMT), using a five-point Likert scale $(1=$ minimum, $5=$ maximum $)$.

Results: A total of 239 physicians completed the questionnaire during 2008-2009. Experience was defined as at least two years of service as military medical personal. There was no significant difference between total attitude scores of experienced and nonexperienced physicians ( $41.7 \pm 5.1$ and $39.0 \pm 0.95, p=0.054$, respectively) and between those $<30$ years of age and $>30$ years $(39.2 \pm 4.7$ and $40.5 \pm 5.0, p=0.1$, respectively).

Discussion: The CBRN training and instructions have a positive impact on attitude of physicians toward management to chemical casualties. This has importance as part of the medical teams preparedness toward a toxicological mass-casualty event, in the battlefield or as terrorist attack. In this sample, there was a dramatic increase in knowledge (31\%). Data regarding the effect of CBRN education showed that Web-based learning showed no increase of knowledge, ${ }^{6}$ thus, it might imply that passive, Web-based education is not enough. Information probably should be transferred by other means such as lectures and hands-on training.

Medical experience had a positive impact on the baseline attitude toward chemical casualties. However, after the training, the non-experienced had a significantly higher increase in attitude score, resulting in similar endpoint scores. This baseline difference was attributed to knowledge and self-perspective regarding capabilities. These findings might demonstrate the importance of training, as it has a positive impact on experienced and non-experienced personnel to achieve similar attitude scores albeit they start on different levels. Interestingly, there was a different effect of age on attitude.

These data exemplify the positive effect of a more elaborated training consisting of both lectures and hands-on training with full protective gear. Attitude scores demonstrated that training has a significantly positive effect on attitude, more prominent on the non-experienced group, and mainly on knowledge and self-perception domains. Although baseline scores were different between age groups and experience groups, at the end of the course, 
scores of different groups were similar, implying that CBRN medical training is an equator of attitude toward a chemical event. This equality of scores might reflect a similar level of preparedness toward managing a chemical warfare casualty indicates the importance of such education and training.

Conclusions: Training such as the described CBRN course has a significant positive impact on attitude of physicians toward treating a chemical victim, mainly on knowledge and self sense of capabilities. Medical experience is a contributor for positive attitude, however, after the CBRN course, attitude scores were similar for all groups.

References

1. Okumura $\mathrm{T}$, Suzuki K, Fukuda A, Kohama A, Takasu N, Ishimatsu S, et al. The Tokyo subway sarin attack: disaster management, Part 1: Community emergency response. Acad Emerg Med 1998;5(6):613-617.

2. Krivoy A, Layish I, Rotman E, Goldberg A, Yehezkelli Y: OP or not OP: the medical challenge at the chemical terrorism scene. Prehosp Disaster Med 2005;20(3):155-158

3. Flowers LK, Mothershead JL, Blackwell TH: Bioterrorism preparedness. II: The community and emergency medical services systems. Emerg Med Clin North $A m$ 2002;20(2):457-476.

4. Chen FM, Hickner J, Fink KS, Galliher JM, Burstin $\mathrm{H}$ : On the front lines: family physicians' preparedness for bioterrorism.J Fam Pract 2002;51(9):745-750.

5. Alexander GC, Wynia MK. Ready and willing? Physicians' sense of preparedness for bioterrorism. Health Aff (Millwood) 2003;22(5):189-197.

6. Chung S, Mandl KD, Shannon M, Fleisher GR. Efficacy of an educational Web site for educating physicians about bioterrorism. Acad Emerg Med 2004;11(2):143-148.

7. Gershon RR, Gemson DH, Qureshi K, McCollum MC. Terrorism preparedness training for occupational health professionals. $J$ Occup Environ Med 2004;46(12):1204-1209.

Keywords: attitude; casualty management; chemical warfare; experience; physician

Prehosp Disaster Med

\section{Terror and Conflicts}

\section{Preparing for the Predictable Surprise of a Terrorist Bombing \\ R. Hunt; I. Ashkenazi; S. Deitchman; B. Dorn;J. Enders; \\ H. Falk; L. Garbarino; V. Kapil; J. Madden; L. Marcus; \\ K. Sarmiento; S. Sasser \\ Centers for Disease Control and Prevention, and the National Preparedness Leadership Initiative: A Joint Program of the Harvard School of Public Health and Harvard's Kennedy School of Government}

Introduction: The US Institute of Medicine reports that, "Explosions are by far the most common cause of casualties associated with terrorism." However, there have been no successful terrorist attacks in the US resulting in mass injuries and deaths since 11 September 2001. While a terrorist bombing is a predictable surprise, medical preparedness for events that have not occurred for eight years pose challenges to motivation and prioritization. This work describes the Centers for Disease Control and Prevention (CDC's) planning strategies to meet those challenges, and programs to disseminate this information.

Methods: These initiatives are designed to acquire, disseminate, and utilize knowledge about clinical and health system challenges in responding to terrorist bombings. This work was accomplished by collaborating with a wide range of partners including: key health system leaders who responded to bombings internationally, non-governmental health organizations, and federal agencies.

Results: Joint meetings of US and international medical responders from New York City, Boston, Washington DC, Chicago, San Francisco, Los Angeles, Delhi, Israel, London, Madrid, Mumbai, and Pakistan were convened to learn about the medical response to terrorist bombings. Terrorist bombing challenges common around the world were identified, including triage, casualty distribution, standards of care, and healthcare system resilience. A course on clinical care of bombing victims, guidance on surge capacity for terrorist bombings, and a bomb injury surveillance tool was developed and disseminated.

Conclusions: The $\mathrm{CDC}$, in cooperation with a wide range of partners, developed and disseminated new knowledge about challenges, and proposed solutions for the medical response to terrorist bombings. This work has applicability in the US and internationally, and to all mass-casualty events. The next steps include developing a health systems terrorist bombing preparedness course, implementing surge capacity guidance, utilizing the bomb injury surveillance tool, and building on successful international collaborations. Keywords: collaboration; international; preparedness; surveillance; terrorism

Prebosp Disaster Med

Program for Psychological First Aid for the Treatment of Acute Stress Reactions in a General Hospital during Rocket Attacks

Eva Farberoff, $M A$; Jolanda Kaddar, PsyD;

Bella Kreinin, $M D$; Gila Hyams, $R N, M A$

Rambam Health Care Campus, Haifa, Israel

Introduction: During the Second Lebanon War, Haifa and Rambam Hospital were under constant rocket attacks. The hospital had to cope with the wounded soldiers and civilians, suffering from physical and/or mental injuries. Simultaneously, the staff was in personal danger and exposed to Secondary Traumatic Stress (STS).The Trauma Unit of Rambam Hospital and the Mental Health Service of the Ministry of Defense requested increased psychological assistance for the wounded. The emergency program was designed to lower stress and to prevent post-traumatic stress symptoms by early psychological intervention.

Methods: The program was developed and directed by the psychological service, coordinated and collaborated with the trauma unit, psychiatric unit, administrative and medical staff of the hospital. Twenty psychologists from the psychiatric department received special training to give bedside psychological first aid (PFA) to physically wounded patients suffering from acute stress reactions (ASR). Training was immediate, ongoing, on location, included weekly lectures, daily group supervision and written manuals. Individual and group sessions were designed for helping staff suffering from STS.

Results: From the 284 hospitalized soldiers and civilians, 112 received PFA in 14 different wards. Psychological evaluation upon release showed lowered stress levels. Few cases were referred to further treatment after release. Staff reported that they felt supported, contained, and helped by the psychological interventions. 